\title{
Maintenance of activities of daily living despite risk from genetic polymorphism in hemodialysis patients under nutritional management who survived an average of 30 years
}

Kaori Sakamoto ${ }^{1}$, Yoshihiko Kanno ${ }^{2 *}$, Mami Hiraoka ${ }^{3}$, Matsuhiko Hayashi ${ }^{4}$, Yoshiko Kontai ${ }^{5}$ and Yasuo Kagawa ${ }^{6}$

\begin{abstract}
Background: Only $4 \%$ of hemodialysis (HD) patients survive over 25 years after their initiation of HD even in Japan. To elucidate their clinical characteristics, we investigated their lifestyle and genetic factor. $\Pi$ genotype of methylenetetrahydrofolate reductase (MTHFR) C677T polymorphism was reported as a high-risk factor for cardiovascular event and poor survival in CKD patients.

Method: Seventy-eight of Japanese patients receiving HD more than 30 years were enrolled. Their daily lifestyle and activity were evaluated with diet history questionnaires (DHQ), geriatric nutritional risk index (GNRI), and basic activity of daily living (BADL) scores. MTHFR C677T was genotyped by PCR-restriction fragment length polymorphism (RFLP).

Results: The mean dietary intake of energy was $30.6 \pm 9.3 \mathrm{kcal} / \mathrm{kg}$ of ideal body weight (IBW), protein $1.1 \pm 0.4 \mathrm{~g} / \mathrm{kg}$ of IBW and their adequacy ratios for Japanese guideline 2007 were 97.7 and $101.9 \%$, respectively. BADL was 90, and daily activities were highly maintained in patients. The frequency of $\Pi$ genotype was $26.9 \%$ and it was almost twice as that in the general population. The patients with $\Pi$ genotype had lower serum folate and higher serum homocysteine than those with the CC or CT genotypes, though there was no significant difference in dietary folate intake among them.
\end{abstract}

Conclusion: Although the frequency of $\Pi$ genotype was higher than healthy population, our patients showed longer survival with high QOL and nutritional status. It is suggested that the proper lifestyle might overcome the genetic risk factors in patients receiving HD.

Keywords: Long survival, Genetic factor, RFLP

\section{Background}

Increasing importance has been attached to the complication of a nutritional disorder in dialysis patients as a risk factor that leads to a poor outcome [1]. Moreover, many dialysis patients are in a protein-energy wasting (PEW) state [2], and this condition has been reported to be a factor that strongly influences the survival of elderly

\footnotetext{
* Correspondence: kannoyh@tokyo-med.ac.jp

${ }^{2}$ Department of Nephrology, Tokyo Medical University, 6-7-1 Nishi-Shinjuku, Shinjuku, Tokyo 160-0023, Japan

Full list of author information is available at the end of the article
}

and long-term dialysis patients [1, 3-5]. The survival rate after introduction of dialysis has been increasing with advances in dialysis techniques in Japan, but the 510 - and 25-year survival rates are only 60.3, 36.2 and $14.1 \%$, respectively, and patients under long-term dialysis treatment for 25 years or longer account for only $4 \%$ (11,802 patients) of the all dialysis patients [6]. "Longterm" outcomes represent outcomes at around 5 years in reports from other countries, and patients on hemodialysis for a short time (less than 10 years) were investigated in most studies in Japan. There have been only a 
few studies in which outcomes and nutritional condition were investigated in patients on hemodialysis for more than 25 years. Moreover, it is not easy to perform a prospective study of long-term outcomes, nutritional condition, and nutritional management. Therefore, it is important to clarify the nutritional condition and food intake of patients who have been receiving hemodialysis for more than 25 years.

In Japan, the causes of death of dialysis patients are heart failure (26.6 \%), infection (20.3\%), malignant tumor $(9.1 \%)$, cerebrovascular disorder (7.7 \%), myocardial infarction (4.4\%), and hyperkalemia (2.9\%). Cardiovascular diseases account for about $40 \%$ of the causes of death and contribute to the poor prognosis of dialysis patients [6]. Especially, ischemic heart disease is a significant obstacle to the maintenance of a favorable quality of life (QOL). Bachmann et al. [7] confirmed that hyperhomocysteinemia is a risk factor for cardiovascular disease in hemodialysis patients. Homocysteine (Hcy) is an amino acid produced through metabolism of an essential amino acid, methionine. Dietary folate-derived 5methyltetrahydrofolate is required for this metabolism as a methyl-group donor. Methylenetetrahydrofolate reductase (MTHFR) catalyzes the irreversible conversion of 5 , 10-methylenetetrahydrofolate to 5-methyltetrahydrofolate. The MTHFR C677T polymorphisms is a $\mathrm{C}$ to $\mathrm{T}$ transition at position 677 (exon4), which causes the substitution of alanine with valine and leads to about $35 \%$ decrease in enzyme activity in CT heterozygotes and $60 \%$ decrease in TT homozygotes [8]. This reduced enzyme activity causes an elevating serum Hcy level [8]. The associations of this polymorphism to hyperhomocysteinemia and cardiovascular disease have been reported. In a study reported in Japan, frequency of $\mathrm{T}$ allele was $33 \%$ and the frequency of TT genotype was $10.2 \%$ in Japanese population, and the T allele frequency was higher in ischemic heart disease and cerebral infarction patients than in normal controls. The serum Hcy level was higher in patients with TT genotype than in those with other genotypes, and the relationship between Hcy and genotype was stronger in the low folate intake [9]. The frequency of the TT genotype was $13.7 \%$ in a study of hemodialysis patients, and it was higher $(23.8 \%)$ in patients with a cardiovascular disorder, showing that the MTHFR polymorphism is an important factor that influences the serum Hcy level [10, 11]. However, there have been only a few studies of Japanese patient with renal failure and hemodialysis patients, and the MTHFR C677T polymorphism has not been investigated in longterm hemodialysis patients.

This study was performed to clarify the actual nutrition state and food intake of long-term hemodialysis patients after more than 25 years of dialysis. We also investigated nutritional management during long-term hemodialysis which is considered as a risk factor for mortality, as association between MTHFR C677T polymorphism, which was based on the risks of renal dysfunction and introduction of dialysis.

\section{Methods \\ Subjects}

Ninety-five patients who had received hemodialysis for more than 25 years or longer were selected from outpatients undergoing hemodialysis three times a week at 14 institutions including Keio University Hospital and related facilities. Documents explaining the objective of the survey and protection of personal information were handed to the subjects, and written consent was obtained from 90 patients after an explanation had been given by physicians. Three patients with missing laboratory test values, one with missing dietary survey result, one with renal transplantation, and one who died during the survey period were excluded. Another six patients were excluded because of extreme under/over-reporting, which was assessed based on the method reported by Sasaki, the developer of brief-type self-administered diet history questionnaire (BDHQ) [12]. The exclusion criteria of the dietary survey are as follows, based on the Dietary Reference Intakes for Japanese, 2010 edition: "an energy intake lower than 0.5 times the estimated energy requirement for physical activity level I, and an energy intake of 1.5 times or higher than the estimated energy requirement for physical activity level III [13]". Subjects in this study finally consisted of 78 patients (31 male and 47 females). The survey period was from March to November in 2012.

This study was approved by Keio University School of Medicine Ethics Committee (approval number 2011-271, dated January 11, 2012) and Experimental Study Ethics Committee of Kagawa Education Institute of Nutrition (approval number 201-G, dated March 14, 2012).

\section{Measurements}

The subject characteristics (sex, age, duration of dialysis therapy, age at the time of introduction of dialysis, and primary disease for renal failure), physical conditions (height, dry weight [DW], and body mass index [BMI]) were assessed. For blood chemistry, blood samples were collected at the beginning of the first dialysis session of the week (Monday or Tuesday) immediately before dialysis, and serum albumin (Alb), blood urea nitrogen (BUN), serum creatinine (Cr), serum potassium (K), serum inorganic phosphorus (IP), serum calcium (Ca), hemoglobin $(\mathrm{Hb})$, hematocrit $(\mathrm{Hct})$, serum folate, serum homocysteine (Hcy), and serum vitamin $\mathrm{B}_{12}\left(\mathrm{VB}_{12}\right)$ were analyzed. The concentrations of serum folate and vitamin $B_{12}$ were measured using chemiluminescent enzyme immunoassay (CLEIA) on UniCel DxI 800 with Access Folate and Access Vitamin B12, respectively (Beckman 
Table 1 Subject characteristics

\begin{tabular}{|c|c|c|}
\hline Variable & & Total $(n=78)$ \\
\hline$\overline{\text { Age }}$ & Years & $632 \pm 8.1^{\mathrm{a}}$ \\
\hline Gender (Male/female) & $n(\%)$ & $31 / 47(39.7 / 60.3 \%)^{b}$ \\
\hline Duration of hemodialysis & Years & $305(27.0-34.3)^{c}$ \\
\hline Age of initiation of hemodialysis & Years & $32.4 \pm 8.0$ \\
\hline \multicolumn{3}{|l|}{ Primary disease } \\
\hline Chronic glomerulonephritis & $n(\%)$ & $55(70.5 \%)$ \\
\hline Chronic pyelonephritis & $n(\%)$ & $1(1.3 \%)$ \\
\hline Nephropathy of pregnancy & $n(\%)$ & $3(3.8 \%)$ \\
\hline Polycystic kidney & $n(\%)$ & $2(2.6 \%)$ \\
\hline $\begin{array}{l}\text { Other nephritides that } \\
\text { cannot be classified }\end{array}$ & $n(\%)$ & $4(5.1 \%)$ \\
\hline Other & $n(\%)$ & $2(2.6 \%)$ \\
\hline Unknown & $n(\%)$ & $11(14.1 \%)$ \\
\hline MTHFR C677T (CC/CT/TT) & n (\%) & $\begin{array}{l}23 / 34 / 21 \\
(29.5 / 43.6 / 26.9 \%)\end{array}$ \\
\hline Height & $\mathrm{cm}$ & $157.6 \pm 8.3$ \\
\hline Weight (dry weight) & $\mathrm{kg}$ & $46.3(41.7-57.0)$ \\
\hline Body mass index (BMI) & $\mathrm{kg} / \mathrm{m}^{2}$ & $197.7 \pm 2.7$ \\
\hline $\mathrm{BMI}<18.5$ & $\begin{array}{l}n \text { (male/female) } \\
(\%)\end{array}$ & $32(7 / 25)(41.0 \%)$ \\
\hline $18.5 \leqq \mathrm{BMI}<25$ & $\begin{array}{l}n \text { (male/female) } \\
(\%)\end{array}$ & $42(21 / 21)(53.8 \%)$ \\
\hline $25 \leqq \mathrm{BMl}$ & $\begin{array}{l}n \text { (male/female) } \\
(\%)\end{array}$ & $4(3 / 1)(5.1 \%)$ \\
\hline Geriatric nutrition index (GNRI) & & $93 \pm 6$ \\
\hline $\begin{array}{l}\geqq \text { GNRI } 91 \text { (without risk } \\
\text { of malnutrition) }\end{array}$ & $n(\%)$ & $46(59.0 \%)$ \\
\hline $\begin{array}{l}<\text { GNRI } 91 \text { (with risk } \\
\text { of malnutrition) }\end{array}$ & $n(\%)$ & $32(41.0 \%)$ \\
\hline Barthel Index (BADL) & & $90.0(78-100)$ \\
\hline $\begin{array}{l}\text { BADL (independent/some } \\
\text { help is necessary/with help) }\end{array}$ & $n(\%)$ & 74/4/3 (91/5/4 \%) \\
\hline $\begin{array}{l}\text { Weight change rate } \\
\text { between dialysis }^{d}\end{array}$ & $\%$ & $5.1 \pm 1.9$ \\
\hline $\mathrm{Kt} / \mathrm{Nsp}{ }^{\mathrm{e}}$ & & $1.67 \pm 0.2$ \\
\hline \multicolumn{3}{|l|}{ Biochemical parameters } \\
\hline Serum albumin (alb) & $\mathrm{g} / \mathrm{dL}$ & $3.70 \pm 0.3$ \\
\hline Total protein (TP) & $\mathrm{g} / \mathrm{dL}$ & $6.60 \pm 0.4$ \\
\hline Blood urea nitrogen (BUN) & $\mathrm{mg} / \mathrm{dL}$ & $65.00 \pm 17.1$ \\
\hline Serum creatinine $(\mathrm{Cr})$ & $\mathrm{mg} / \mathrm{dL}$ & $10.70 \pm 2.2$ \\
\hline Urea acid (UA) & $\mathrm{mg} / \mathrm{dL}$ & $7.10 \pm 1.5$ \\
\hline Serum sodium (Na) & $\mathrm{mEq} / \mathrm{L}$ & $140.00 \pm 2.5$ \\
\hline Serum potassium (K) & $\mathrm{mEq} / \mathrm{L}$ & $5.10 \pm 0.7$ \\
\hline $\begin{array}{l}\text { Serum inorganic } \\
\text { phosphorous (IP) }\end{array}$ & $\mathrm{mg} / \mathrm{dL}$ & $5.40 \pm 1.4$ \\
\hline Serum calcium (Ca) & $\mathrm{mg} / \mathrm{dL}$ & $9.10 \pm 0.7$ \\
\hline Hemoglobin ( $\mathrm{Hb})$ & $\mathrm{g} / \mathrm{dL}$ & $10.30(9.6-11.2)$ \\
\hline Hematocrit (Hb) & $\%$ & $33.00 \pm 3.4$ \\
\hline
\end{tabular}

Table 1 Subject characteristics (Continued)

\begin{tabular}{|c|c|c|}
\hline Serum folate & $\mathrm{ng} / \mathrm{mL}$ & $4.60(3.8-5.4)$ \\
\hline Serum homocystein (Hcy) & $\mu \mathrm{mol} / \mathrm{L}$ & $30.80(23.6-38.4)$ \\
\hline Serum vitamin in $\mathrm{B} 12\left(\mathrm{VB}_{12}\right)$ & $\mathrm{pmol} / \mathrm{L}$ & $541.00(340-1250)$ \\
\hline \multicolumn{3}{|c|}{ 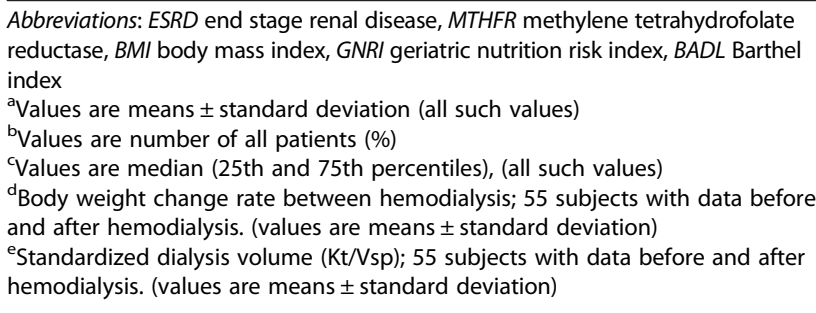 } \\
\hline
\end{tabular}

Coulter). Serum Hcy concentrations were measured by high-performance liquid chromatography (HPLC). These measurements before and after dialysis were available in 55 patients, and the index of dialysis efficiency, Kt/Vsp, was calculated using Shinzato's formula [14]. Blood chemistry was analyzed by SRL Tokyo Medical (Tokyo, Japan).

Habitual energy, nutrient, and food group intakes were surveyed using BDHQ $[15,16]$ which is a simplified version of the self-administered diet history questionnaire (DHQ) developed by Sasaki et al. [17]. To assess activities of daily living, basic activities of daily living were evaluated using BADL published by Mahoney and Barthel in 1965 [18]. The geriatric nutrition risk index (GNRI) used for nutrition screening of dialysis patients was calculated using the formula below. GNRI is a nutrition screening tool for the elderly developed by Bouillanne et al. [19]. Yamada et al. [20] applied GNRI in a study of dialysis patients and reported its usefulness.

DNA was extracted from EDTA-added whole-blood samples collected as described above using a fully automated nucleic acid extraction device, Magtration ${ }^{\bullet}$ System 6GC, and a reagent that is used exclusively with this device, MagDEA DNA 200 (GC) (Precision System Science Co., Ltd., Japan). The MTHFR C677T polymorphism was genotyped using polymerase chain reaction (PCR) technique and restriction fragment length polymorphism (RFLP) analysis by PaGE (Tokyo, Japan). The patients were divided into three groups based on the MTHFR C677T genotype, and the association with each index (physical condition, laboratory test values, food intake, daily living activities, and GNRI) was investigated.

\section{Statistical analysis}

Variables detected in the measurements and survey items were confirmed using the Shapiro-Wilk test of normality and histograms. When the distribution was not normal, logarithmic transformation or nonparametric test was employed. Log-transformed serum Hcy was examined using linear regression analysis. Data sets of variables with normality were presented as means \pm 
Table 2 Habitual dietary intakes

\begin{tabular}{|c|c|c|}
\hline Variable & & Total $(n=78)$ \\
\hline \multicolumn{3}{|l|}{ Intakes of nutrients and energy } \\
\hline Energy & kcal & 1635 (1247-1993) \\
\hline Energy & $\mathrm{kcal} / \mathrm{kglBW}$ & $29.2(22.8-35.8)$ \\
\hline Protein & $g$ & $57.6(44.0-72.7)$ \\
\hline Protein & $\mathrm{g} / \mathrm{kglBW}$ & $1.1(0.8-1.3)$ \\
\hline Ratio of animal protein & $\%$ & $56.2(46.1-64.0)$ \\
\hline $\mathrm{NPC} / \mathrm{N}^{a}$ & & $147(126-175)$ \\
\hline Fat & $\% \mathrm{E}$ & $27.5(22.5-30.7)$ \\
\hline Carbohydrate & $\% \mathrm{E}$ & $56.6(51.6-61.1)$ \\
\hline Retinol & $\mu \mathrm{g}$ & $556(398-878)$ \\
\hline a-tocopherol & $\mathrm{mg}$ & $6.7(5.1-8.7)$ \\
\hline Potassium & $\mathrm{mg}$ & $1946(1463-2586)$ \\
\hline Calcium & $\mathrm{mg}$ & $386(248-502)$ \\
\hline Phosphorus & $\mathrm{mg}$ & $841(609-1062)$ \\
\hline Iron & $\mathrm{mg}$ & $6.6(4.6-8.3)$ \\
\hline Vitamin $_{6}$ & $\mathrm{mg}$ & $1.0(0.7-1.3)$ \\
\hline Vitamin $_{12}$ & $\mu g$ & $7.1(4.6-10.1)$ \\
\hline Folate & $\mu \mathrm{g}$ & $285(200-370)$ \\
\hline VitaminC & $\mathrm{mg}$ & $99(71-136)$ \\
\hline Salt & g & $8.9(7.4-10.8)$ \\
\hline \multicolumn{3}{|l|}{ Energy-adjusted nutrient intakes } \\
\hline Retinol & $\mu \mathrm{g} / 1000 \mathrm{kcal}$ & $350(255-490)$ \\
\hline a-tocopherol & $\mathrm{mg} / 1000 \mathrm{kcal}$ & $4.2(3.6-4.9)$ \\
\hline Potassium & $\mathrm{mg} / 1000 \mathrm{kcal}$ & $1211(1021-1469)$ \\
\hline Calcium & $\mathrm{mg} / 1000 \mathrm{kcal}$ & $225(179-294)$ \\
\hline Phosphorus & $\mathrm{mg} / 1000 \mathrm{kcal}$ & $523(453-599)$ \\
\hline Iron & $\mathrm{mg} / 1000 \mathrm{kcal}$ & $4.0(3.4-4.6)$ \\
\hline Vitamin $_{6}$ & mg/1000 kcal & $0.6(0.5-0.7)$ \\
\hline$V_{i t a m i n} B_{12}$ & $\mu \mathrm{g} / 1000 \mathrm{kcal}$ & $4.5(3.2-6.4)$ \\
\hline Folate & $\mu \mathrm{g} / 1000 \mathrm{kcal}$ & $169(137-219)$ \\
\hline VitaminC & $\mathrm{mg} / 1000 \mathrm{kcal}$ & $64(44-77)$ \\
\hline Salt & $\mathrm{g} / 1000 \mathrm{kcal}$ & $5.5(4.9-6.3)$ \\
\hline \multicolumn{3}{|l|}{ Energy-adjusted food group intakes } \\
\hline Cereals & $\mathrm{g} / 1000 \mathrm{kcal}$ & $220(185-297)$ \\
\hline Potatoes & $\mathrm{g} / 1000 \mathrm{kcal}$ & $18(8-43)$ \\
\hline Sugar and confectioneries & $\mathrm{g} / 1000 \mathrm{kcal}$ & $2.9(1.7-3.7)$ \\
\hline Nuts and pulses & $\mathrm{g} / 1000 \mathrm{kcal}$ & $18(8-28)$ \\
\hline Green and yellow vegetables & $\mathrm{g} / 1000 \mathrm{kcal}$ & $52(32-79)$ \\
\hline Other vegetables & $\mathrm{g} / 1000 \mathrm{kcal}$ & $68(49-101)$ \\
\hline Fruits & $\mathrm{g} / 1000 \mathrm{kcal}$ & $43(18-85)$ \\
\hline Fish and shellfish & $\mathrm{g} / 1000 \mathrm{kcal}$ & $39(24-54)$ \\
\hline Meat & $\mathrm{g} / 1000 \mathrm{kcal}$ & $35(25-52)$ \\
\hline Eggs & $\mathrm{g} / 1000 \mathrm{kcal}$ & $18(7-31)$ \\
\hline Daily products & $\mathrm{g} / 1000 \mathrm{kcal}$ & $24(7-64)$ \\
\hline
\end{tabular}

Table 2 Habitual dietary intakes (Continued)

\begin{tabular}{lcc}
\hline Fats and oils & $\mathrm{g} / 1000 \mathrm{kcal}$ & $6.4 \mathrm{~b}(4.4-8.6)$ \\
$\begin{array}{l}\text { Confectioneries } \\
\text { Alcoholic beverages and }\end{array}$ & $\mathrm{g} / 1000 \mathrm{kcal}$ & $24(13-50)$ \\
non-alcoholic beverages & $\mathrm{g} / 1000 \mathrm{kcal}$ & $212(138-336)$ \\
$\begin{array}{l}\text { Seasoning and spice } \\
\text { All values are median (25-75percentiles) }\end{array}$ & $81(53-139)$ \\
${ }^{\text {NNPC/N, Ratio of non-protein energy/nitrogen }}$ &
\end{tabular}

standard deviations and those without normality were presented as medians (25th and 75th percentiles). Regarding food intake, the measured energy intake, animal protein ratio, lipid energy ratio, iron intake, and energyadjusted potassium and $\mathrm{B}_{6}$ intakes showed normal distributions, but the others did not. Since the median and mean are similar when the distribution is normal, all data were presented as medians (25th and 75th percentiles).

In the comparison of the three MTHFRC 677T genotype-based groups (CC, CT, and TT groups), the interval and ratio scales were compared using one-way analysis of variance and multiple comparison (homogeneous variance: Tukey's test; non-homogeneous variance: Games-Howell test), or the Kruskal-Wallis test and multiple comparison (Bonferroni correction; Mann-Whitney $U$ test). For the nominal scale, the $\chi^{2}$ test was employed.

Statistical analysis was performed using a statistical package, IBM SPSS Statistics Ver. 20 (IBM, Tokyo), and the significance level was set at less than $5 \%$. When a missing value was present, the item was deleted entirely.

\section{Results \\ Subject characteristics}

1. Clinical profile

The characteristics, physical condition, and biochemical parameters of the study subjects were shown in Table 1.

The mean score of the nutritional disorder risk index, GNRI, was $93 \pm 6$, and the percentage of patients with a nutritional disorder risk with a GNRI score of less than 91 was $41.0 \%$.

2. Habitual dietary intakes

The median (25th and 75th percentiles) daily habitual energy, nutrient, energy-adjusted nutrient, and food group intakes of the patients are shown in Table 2.

3. Adequacy ratio for "Dietary recommendations for chronic kidney disease (CKD), 2007 (guidelines) [21]". The energy, protein, potassium, and phosphorus intake sufficiency rates were 97.7, 101.9, 103.6, and $96.7 \%$, respectively, which met the recommendations of the guidelines, but the salt intake sufficiency rate was $152.6 \%$, indicating excessive ingestion, and only $12.8 \%$ of patients met the recommendation of less than $6 \mathrm{~g} /$ day. 
Table 3 Clinical characteristics of patients (MTHFR C677T genotype)

\begin{tabular}{|c|c|c|c|c|c|}
\hline \multirow{3}{*}{ Variable } & & \multicolumn{4}{|l|}{ Genotype } \\
\hline & & \multirow{2}{*}{$\begin{array}{l}\text { CC } \\
(n=23) 29.5 \%\end{array}$} & \multirow{2}{*}{$\begin{array}{l}\mathrm{CT} \\
(n=34) 43.6 \%\end{array}$} & \multirow{2}{*}{$\begin{array}{l}\text { TT } \\
(n=21) 26.9 \%\end{array}$} & \multirow[b]{2}{*}{$P$ value } \\
\hline & & & & & \\
\hline Age & years & $62.7 \pm 6.9^{a}$ & $63.6 \pm 8.7$ & $63.2 \pm 8.7$ & 0.920 \\
\hline Gender(Male/Female) & $\mathrm{n}$ & $11 / 12$ & $10 / 29$ & $10 / 11$ & 0.261 \\
\hline Duration of hemodialysis & years & $28.0(25.0-31.0)^{\mathrm{b}}$ & $31(27.0-34.3)$ & $32.0(28.5-35.0)$ & 0.276 \\
\hline Age at initiation of hemodialysis & years & $33.0 \pm 7.3$ & $32.7 \pm 8.5$ & $31.3 \pm 8.2$ & 0.760 \\
\hline Height & $\mathrm{cm}$ & $159.7 \pm 9.1$ & $155.7 \pm 8.1$ & $158.5 \pm 7.5$ & 0.170 \\
\hline Dry weight(DW) & $\mathrm{kg}$ & $50.0^{\mathrm{d}}(43.8-59.5)$ & $44.0^{\mathrm{e}}(39.1-50.9)$ & $52.6^{d}(44.5-59.3)$ & $0.014^{*}$ \\
\hline Body mass index(BMI) & $\mathrm{kg} / \mathrm{m}^{2}$ & $20.2 \pm 19.7$ & $18.8 \pm 2.8$ & $20.5 \pm 2.6$ & 0.050 \\
\hline $\mathrm{BMI}<18.5$ & n (\%) & $7(21.9 \%)^{c}$ & 19 (59.4\%) & $6(18.8 \%)$ & \\
\hline $18.5 \leqq \mathrm{BMl}<25$ & n (\%) & $15(35.7 \%)$ & 14(33.3\%) & $13(31.0 \%)$ & \\
\hline $25 \leqq \mathrm{BMl}$ & n (\%) & $1(25.0 \%)$ & $1(25.0 \%)$ & $2(50.0 \%)$ & \\
\hline Bathel Index(BADL) & & $90(74-100)$ & $98(84-100)$ & $85(78-100)$ & 0.253 \\
\hline BADL(independent/some help is necessary/with help) & $\mathrm{n}$ & $20 / 2 / 1$ & $33 / 1 / 0$ & $18 / 1 / 2$ & $<0.001^{*}$ \\
\hline Geriatric nutrition risk index(GNRI) & & $9489-90$ & $9088-96$ & $9491-99$ & 0.200 \\
\hline$\geqq G N R I 91$ (without risk of malnutrition) & $n(\%)$ & $16(34.8 \%)$ & $15(32.6 \%)$ & $15(32.6 \%)$ & \\
\hline$<$ GNRI 91 (with risk of malnutrition) & $n(\%)$ & $7(21.9 \%)$ & $19(59.4 \%)$ & $6(18.8 \%)$ & \\
\hline \multicolumn{6}{|l|}{ Biochemical parameter } \\
\hline serum albumin (Alb) & $\mathrm{g} / \mathrm{dL}$ & $3.7 \pm 0.3$ & $3.8 \pm 0.3$ & $3.7 \pm 0.3$ & 0.887 \\
\hline total protein (TP) & $\mathrm{g} / \mathrm{dL}$ & $6.4^{d} \pm 0.3$ & $6.6 \pm 0.5$ & $6.7^{e} \pm 0.5$ & $0.045^{*}$ \\
\hline blood urea nitrogen (BUN) & $\mathrm{mg} / \mathrm{dL}$ & $63.8 \pm 14$ & $64.2 \pm 6.6$ & $67.6 \pm 21.2$ & 0.718 \\
\hline serum creatinine $(\mathrm{Cr})$ & $\mathrm{mg} / \mathrm{dL}$ & $10.8 \pm 2.3$ & $10.5 \pm 2.2$ & $10.8 \pm 2.2$ & 0.873 \\
\hline urea acid (UA) & $\mathrm{mg} / \mathrm{dL}$ & $7.1 \pm 1.3$ & $6.9 \pm 1.7$ & $7.5 \pm 1.5$ & 0.341 \\
\hline serum sodium $(\mathrm{Na})$ & $\mathrm{mEq} / \mathrm{L}$ & $141 \pm 2$ & $140 \pm 2.6$ & $140 \pm 2.9$ & 0.847 \\
\hline serum potassium (K) & $\mathrm{mEq} / \mathrm{L}$ & $5.1 \pm 0.6$ & $5.2 \pm 0.7$ & $5.1 \pm 0.6$ & 0.864 \\
\hline serum inorganic phosphorus (IP) & $\mathrm{mg} / \mathrm{dL}$ & $5.7 \pm 1.1$ & $5.1 \pm 1.2$ & $5.6 \pm 1.8$ & 0.164 \\
\hline serum calcium (Ca) & $\mathrm{mg} / \mathrm{dL}$ & $8.9 \pm 0.7$ & $9.3 \pm 0.7$ & $9 \pm 0.6$ & 0.096 \\
\hline Hemoglobin ( $\mathrm{Hb})$ & $\mathrm{g} / \mathrm{dL}$ & $10.4(9.1-11.5)$ & $10.3(10.1-11.7)$ & $10.2(9.5-11.1)$ & 0.789 \\
\hline Hematocrit $(\mathrm{Ht})$ & $\%$ & $33.5 \pm 4.3$ & $32.9 \pm 3.3$ & $32.7 \pm 2.9$ & 0.164 \\
\hline serum folate & $\mathrm{ng} / \mathrm{mL}$ & $4.7^{d}(4.3-5.6)$ & $4.7^{d}(3.8-6.0)$ & $3.9^{\mathrm{e}}(3.2-4.5)$ & $0.005^{*}$ \\
\hline serum homocystein (Hcy) & $\mu \mathrm{mol} / \mathrm{L}$ & $30.5^{d}(25.2-37.6)$ & $25.1^{d}(22.1-36.9)$ & $35.4^{\mathrm{e}}(27.3-58.3)$ & $0.029^{*}$ \\
\hline serum vitamin $B_{12}\left(\mathrm{VB}_{12}\right)$ & $\mathrm{pmol} / \mathrm{L}$ & $545(314-1200)$ & $630(383-1328)$ & $456(291-1463)$ & 0.763 \\
\hline
\end{tabular}

Abbreviations: MTHFR Methylene tetrahydrofolate reductase, $B M I$ body mass index, GNRI geriatric nutrition risk index, $B A D L$ Barthel index

${ }^{a}$ Values are means \pm Standard deviation(all such values)

balues are median(25th and 75th percentiles)(all such values)

'Values are number of all patients (\%)

The normality of the data was first assessed using the Shapiro-Wilks test

The values are compared between the groups by the $x^{2}$ test, one-way analysis of variance and Tukey's multiple comparison test of or Games-Howell's test, and Kruskal-Wallis test and Bonferroni's multiple comparison test as appropriate. ${ }^{*} p<0.05$

${ }^{d-e}$ Multiple comparison; A different alphabet shows that there is a significant difference

4. Frequency of genetic polymorphism

The frequencies of MTHFR C677T genotype were 29.5, 43.6, and $26.9 \%$ for CC, CT, and TT genotypes, respectively. This showed that the frequency of TT type was significantly higher than that in Japanese hemodialysis patients as reported by Morimoto et al. (13.7\%) [11] and Kimura et al. $(17.4 \%)[22](P<0.05)$, and this was also significantly higher than that in healthy Japanese (about $15 \%)[23,24](P<0.05)$.

5. Activities of daily living

The median score of BADL, which evaluates the performance of activities of daily living, was 90 (range, 78-100). Notably, $91 \%$ of subjects were "independent" while $5 \%$ "required partial assistance" and $4 \%$ "required assistance". 
Table 4 Nutrient intakes and food group intakes of subjects with 3 different genotype of MTHFR C677T polymorphism

\begin{tabular}{|c|c|c|c|c|c|c|c|c|}
\hline \multirow{2}{*}{$\begin{array}{l}\text { Variable } \\
\text { Energy }\end{array}$} & \multirow[b]{2}{*}{ kcal } & \multicolumn{2}{|c|}{$C C(n=23) 29.5 \%$} & \multicolumn{2}{|c|}{$\mathrm{CT}(n=34) 43.6 \%$} & \multicolumn{2}{|c|}{$\Pi(n=21) 26.9 \%$} & \multirow{2}{*}{$\frac{P \text { value }}{0.088}$} \\
\hline & & 1897 & $(1299-2283)$ & 1452 & (1158-1812) & 1584 & $(1365-1945)$ & \\
\hline Energy & $\mathrm{kcal} / \mathrm{kglBW}$ & 33.0 & $(23.5-40.0)$ & 27.6 & $(21.6-34.7)$ & 30 & $(23.7-34.6)$ & 0.155 \\
\hline Protein & $g$ & $62.2^{\mathrm{b}}$ & $(49.4-85.2)$ & $49.7^{c}$ & $(38.5-66.6)$ & 58.9 & $(42.6-72.6)$ & $0.036^{*}$ \\
\hline Protein & $\mathrm{g} / \mathrm{kglBW}$ & 1.1 & $(1.0-1.6)$ & 0.9 & $(0.8-1.3)$ & 1.1 & $(0.8-1.2)$ & 0.086 \\
\hline ratio of animal protein & $\%$ & 58 & $(52-63)$ & 54 & $(42-66)$ & 55 & $(43-63)$ & 0.332 \\
\hline $\mathrm{NPC} / \mathrm{N}^{\mathrm{a}}$ & & 144 & (126-154) & 147 & $(129-191)$ & 152 & $(120-200)$ & 0.383 \\
\hline Fat & $\% \mathrm{E}$ & 28.5 & $(26.2-33.2)$ & 27 & $(21.5-30.5)$ & 23.7 & $(19.5-29.8)$ & 0.067 \\
\hline Carbohydrate & $\% \mathrm{E}$ & 54.8 & $(51.7-57.2)$ & 57.6 & $(50.7-65.8)$ & 57.9 & $(54.0-64.3)$ & 0.210 \\
\hline Retinol & $\mu g$ & 620 & $(457-1143)$ & 538 & $(328-791)$ & 552 & (371-828) & 0.184 \\
\hline a-tocopherol & $\mathrm{mg}$ & $8.2^{b}$ & $(6.0-10.4)$ & $5.8^{c}$ & $(4.3-7.4)$ & 6.8 & $(5.0-8.7)$ & $0.025^{*}$ \\
\hline Potassium & $\mathrm{mg}$ & 2069 & $(1539-2945)$ & 1802 & $(1359-2185)$ & 2104 & $(1541-2618)$ & 0.208 \\
\hline Calcium & $\mathrm{mg}$ & 398 & $(290-573)$ & 373 & $(230-473)$ & 386 & $(249-476)$ & 0.399 \\
\hline Phosphorus & $\mathrm{mg}$ & 987 & $(677-1212)$ & 725 & (577-998) & 847 & $(657-1048)$ & 0.073 \\
\hline Iron & $\mathrm{mg}$ & 7.7 & $(5.7-9.0)$ & 6.0 & $(4.2-8.1)$ & 6.5 & $(4.2-8.1)$ & 0.114 \\
\hline Vitamin $B_{6}$ & $\mathrm{mg}$ & 1.0 & $(0.8-1.5)$ & 0.9 & $(0.7-1.2)$ & 1.0 & $(0.8-1.5)$ & 0.157 \\
\hline$V_{i t a m i n} B_{12}$ & $\mu \mathrm{g}$ & 8.4 & $(6.7-12.2)$ & 6.5 & $(3.8-9.8)$ & 5.4 & $(4.3-11.2)$ & 0.123 \\
\hline Folate & $\mu \mathrm{g}$ & 285 & $(219-436)$ & 279 & $(183-364)$ & 285 & $(183-368)$ & 0.465 \\
\hline VitaminC & $\mathrm{mg}$ & 100 & $(73-166)$ & 93 & $(71-119)$ & 103 & $(51-137)$ & 0.475 \\
\hline Salt & $g$ & $10.3^{\mathrm{b}}$ & $(8.7-12.0)$ & $7.6^{c}$ & $(6.4-9.7)$ & $9.3^{d}$ & (7.8-11.3) & $0.001^{*}$ \\
\hline Retinol & $\mu \mathrm{g} / 1000 \mathrm{kcal}$ & 366 & $(237-556)$ & 350 & $(266-492)$ & 329 & $(235-461)$ & 0.519 \\
\hline a-tocopherol & mg/1000 kcal & 4.3 & $(3.9-5.1)$ & 4.2 & $(3.5-4.8)$ & 4.2 & $(3.2-5.3)$ & 0.476 \\
\hline Potassium & mg/1000 kcal & 1220 & (1014-1373) & 1211 & $(1075-1469)$ & 1210 & (936-1558) & 0.992 \\
\hline Calcium & mg/1000 kcal & 250 & $(185-292)$ & 227 & $(184-300)$ & 222 & $(164-292)$ & 0.359 \\
\hline Phosphorus & $\mathrm{mg} / 1000 \mathrm{kcal}$ & 543 & $(496-588)$ & 516 & $(434-628)$ & 486 & $(405-647)$ & 0.614 \\
\hline Iron & mg/1000 kcal & 4 & $(3.5-4.5)$ & 4.3 & $(3.6-4.6)$ & 3.7 & $(3.0-4.7)$ & 0.407 \\
\hline$V_{\text {Vitamin }}{ }_{6}$ & mg/1000 kcal & 0.6 & $(0.50-0.70)$ & 0.7 & $(0.50-0.73)$ & 0.6 & $(0.50-0.80)$ & 0.969 \\
\hline$V_{i t a m i n} B_{12}$ & $\mu \mathrm{g} / 1000 \mathrm{kcal}$ & 4.7 & $(4.0-5.7)$ & 4.5 & $(3.0-6.2)$ & 4.2 & $(2.7-6.7)$ & 0.609 \\
\hline Folate & $\mu \mathrm{g} / 1000 \mathrm{kcal}$ & 165 & $(138-219)$ & 190 & $(153-223)$ & 164 & $(113-212)$ & 0.445 \\
\hline VitaminC & mg/1000 kcal & 60 & $(47-81)$ & 64 & $(49-77)$ & 65 & $(32-70)$ & 0.808 \\
\hline Salt & $\mathrm{g} / 1000 \mathrm{kcal}$ & 5.7 & $(5.2-6.4)$ & 5.3 & $(4.5-6.2)$ & 5.4 & $(4.4-6.5)$ & 0.164 \\
\hline Cereals & $\mathrm{g} / 1000 \mathrm{kcal}$ & 201 & $(184-284)$ & 220 & $(187-297)$ & 230 & $(185-318)$ & 0.638 \\
\hline Potatos & $\mathrm{g} / 1000 \mathrm{kcal}$ & 12 & $(7-44)$ & 17 & $(7-41)$ & 31 & $(10-53)$ & 0.303 \\
\hline Sugar and confectioneries & $\mathrm{g} / 1000 \mathrm{kcal}$ & 3.5 & $(1.8-4.5)$ & 2.3 & $(1.6-3.2)$ & 3.2 & $(1.7-3.6)$ & 0.149 \\
\hline Nuts and pulses & $\mathrm{g} / 1000 \mathrm{kcal}$ & 19 & $(11-29)$ & 19 & $(11-29)$ & 16 & $(6-29)$ & 0.522 \\
\hline Green and yellow vegetables & $\mathrm{g} / 1000 \mathrm{kcal}$ & 51 & $(27-65)$ & 62 & $(42-83)$ & 49 & $(26-78)$ & 0.343 \\
\hline Other vegetables & $\mathrm{g} / 1000 \mathrm{kcal}$ & 65 & $(49-100)$ & 73 & $(44-101)$ & 80 & $(49-119)$ & 0.933 \\
\hline Fruits & $\mathrm{g} / 1000 \mathrm{kcal}$ & 51 & $(32-107)$ & 32 & $(15-67)$ & 50 & $(17-86)$ & 0.197 \\
\hline Fish and shellfish & $\mathrm{g} / 1000 \mathrm{kcal}$ & 44 & $(26-51)$ & 37 & $(21-52)$ & 41 & $(20-60)$ & 0.717 \\
\hline Meat & $\mathrm{g} / 1000 \mathrm{kcal}$ & 42 & $(29-70)$ & 31 & $(18-50)$ & 36 & $(25-47)$ & 0.087 \\
\hline Eggs & $\mathrm{g} / 1000 \mathrm{kcal}$ & $24^{b}$ & $(11-35)$ & 17 & $(6-28)$ & $8^{c}$ & $(4-21)$ & $0.028^{*}$ \\
\hline Daily products & $\mathrm{g} / 1000 \mathrm{kcal}$ & 28 & $(9-47)$ & 25 & $(8-73)$ & 16 & $(0-61)$ & 0.380 \\
\hline Fats and oils & $\mathrm{g} / 1000 \mathrm{kcal}$ & 7.5 & $(5.4-9.7)$ & 5 & $(3.7-7) 8$. & 6.5 & $(5.8-8.7)$ & 0.055 \\
\hline
\end{tabular}


Table 4 Nutrient intakes and food group intakes of subjects with 3 different genotype of MTHFR C677T polymorphism (Continued)

\begin{tabular}{|c|c|c|c|c|c|c|c|c|}
\hline confectioney & $\mathrm{g} / 1000 \mathrm{kcal}$ & 23 & $(12-46)$ & 24 & $(12-52)$ & 24 & $(14-48)$ & 0.962 \\
\hline Alcoholic bevarages and Non-Alcoholic bevarage & $\mathrm{g} / 1000 \mathrm{kcal}$ & 176 & $(114-304)$ & 207 & $(143-408)$ & 246 & $(154-399)$ & 0.401 \\
\hline seasoning and spice & $\mathrm{g} / 1000 \mathrm{kcal}$ & 81 & $(61-150)$ & 82 & $(51-135)$ & 61 & $(36-121)$ & 0.281 \\
\hline
\end{tabular}

Abbreviations: MTHFR methylenetetrahydrofolate reductase

${ }^{a} N P C / N$ ratio of non-protein energy per nitroden

All values are median (25th and 75th percentiles)

The normality of the data was first assessed using the Shapiro-Wilks test

The values are compared between the groups by Kruskal-Wallis test and Bonferroni's multiple comparison test as appropriate. ${ }^{*} p<0.05$

b-c Multiple comparison; A different alphabet shows that there is a significant difference

\section{Comparison of the genotypes of MTHFR C677T polymorphism}

The characteristics of patients, the physical condition, biochemical parameters and dietary intake, according the MTHFR C677T genotypes (CC, CT, and TT), are shown in Tables 3 and 4. No significant differences were observed in age, duration of dialysis therapy, age at the introduction of dialysis, physical condition, BADL or GNRI among the different genotypes. There were significant differences in TP $(P=0.045)$, serum folate $(P=0.005)$, and serum Hcy $(P=0.029)$, among the genotypes. The serum folate level was significantly lower and the serum Hcy level was significantly higher in the subjects with TT genotype than in those $\mathrm{CC}$ and $\mathrm{CT}$ genotypes. No significant differences were present in serum Alb, BUN, Cr, K, IP, Hb, Ht, and $\mathrm{VB}_{12}$ among the genotypes.

Regarding dietary intake, no significant differences were observed in the energy or protein intake per $\mathrm{kg}$ IBW, animal protein ratio, NPC/N, lipid energy ratio, or carbohydrate energy ratio, nor were there significant differences in the energy-adjusted potassium, phosphorus, folate, vitamin $B_{6}$, or $B_{12}$ intake among the genotypes.

\section{Associations between the serum Hcy level and physical condition, biochemical parameters, and food intakes}

The results of analysis of correlations between the serum Hcy level and physical condition, biochemical parameters and food intake are shown in Tables 5 and 6. No correlation was observed between the serum Hcy level and age, duration of dialysis therapy, BMI or BADL. A positive significant correlation was noted with GNRI $(r=0.33, P=0.003)$. Regarding the association with biochemical parameters, positive correlations were noted with serum Alb $(r=0.271, P=0.009)$ and IP $(r=$ 0.334, $P=0.002$ ) levels, and inverse correlations were noted with serum folate $(r=-0.384, P<0.001)$ and serum $\mathrm{VB}_{12}(r=-0.495, P<0.001)$ levels. Regarding the association with food intake, inverse correlations were noted with animal protein ratio $(r=-0.252, P=0.013)$, energyadjusted vitamin $\mathrm{B}_{6}$ intake $(r=-0.192, p=0.048)$, and $\mathrm{B}_{12}$ intake $(r=-0.242, P=0.017)$. No correlation was noted with energy-adjusted food group intakes.

\section{Factors that may influence the serum Hcy level}

The serum Hcy level was analyzed after testing the normality of the variable using the Shapiro-Wilk test and confirming the distribution by examining the histogram, followed by logarithmic transformation. To investigate factors influencing the serum Hcy level, the correlation matrix was examined with serum Hcy level as a response variable, but no variable with $|r|>0.9$ was present. Thus, multiple regression analysis was performed employing the stepwise method, mainly regarding factors with

Table 5 Pearson's correlation coefficients between serum homocystein and laboratory test values

\begin{tabular}{|c|c|c|c|}
\hline & & & $(n=77)$ \\
\hline & & $r$ & $P$ value \\
\hline Age & year & -0.112 & 0.382 \\
\hline Gender & & -0.237 & $0.038^{*}$ \\
\hline Duration of hemodialysis & year & -0.072 & 0.568 \\
\hline Age at initiation of hemodialysis & year & -0.074 & 0.524 \\
\hline MTHFR C677T & & -0.249 & $0.029^{*}$ \\
\hline Height & $\mathrm{cm}$ & 0.179 & 0.119 \\
\hline Weight(dry weight) & $\mathrm{kg}$ & 0.205 & 0.073 \\
\hline BMl & $\mathrm{kg} / \mathrm{m}^{2}$ & 0.139 & 0.227 \\
\hline GNRI & & 0.330 & $0.003^{*}$ \\
\hline $\mathrm{BADL}$ & & 0.035 & 0.760 \\
\hline serum albumin & $\mathrm{g} / \mathrm{dL}$ & 0.271 & $0.017^{*}$ \\
\hline blood urea nitrogen (BUN) & $\mathrm{mg} / \mathrm{dL}$ & 0.040 & 0.732 \\
\hline serum creatinine $(\mathrm{Cr})$ & $\mathrm{mg} / \mathrm{dL}$ & 0.200 & 0.081 \\
\hline urea acid (UA) & $\mathrm{mg} / \mathrm{dL}$ & 0.211 & 0.065 \\
\hline serum sodium ( $\mathrm{Na}$ ) & $\mathrm{mEq} / \mathrm{L}$ & 0.081 & 0.486 \\
\hline serum potassium (K) & $\mathrm{mEq} / \mathrm{L}$ & 0.026 & 0.822 \\
\hline serum inorganic phosphorus (IP) & $\mathrm{mg} / \mathrm{dL}$ & 0.334 & $0.003^{*}$ \\
\hline serum calcium (Ca) & $\mathrm{mg} / \mathrm{dL}$ & -0.088 & 0.445 \\
\hline Hemoglobin ( $\mathrm{Hb})$ & $\mathrm{g} / \mathrm{dL}$ & 0.071 & 0.541 \\
\hline Hematocrit $(\mathrm{Ht})$ & $\%$ & -0.025 & 0.830 \\
\hline serum folate & $\mathrm{ng} / \mathrm{mL}$ & -0.384 & $0.001^{*}$ \\
\hline serum homocystein (Hcy) & $\mathrm{pmol} / \mathrm{L}$ & -0.495 & $<0.001^{*}$ \\
\hline
\end{tabular}

Abbreviations: MTHFR methylenetetrahydrofolate reductase, $B M I$ body mass index, GNRI geriatric nutrition risk index, $B A D L$ Barthel index

Data not regularly distributed were log transformed for futher statistical analysis. ${ }^{*} P<0.05$

Adjusted for gender, age 
Table 6 Peason's correlation coefficients between serum homocystein consentration and dietary intake

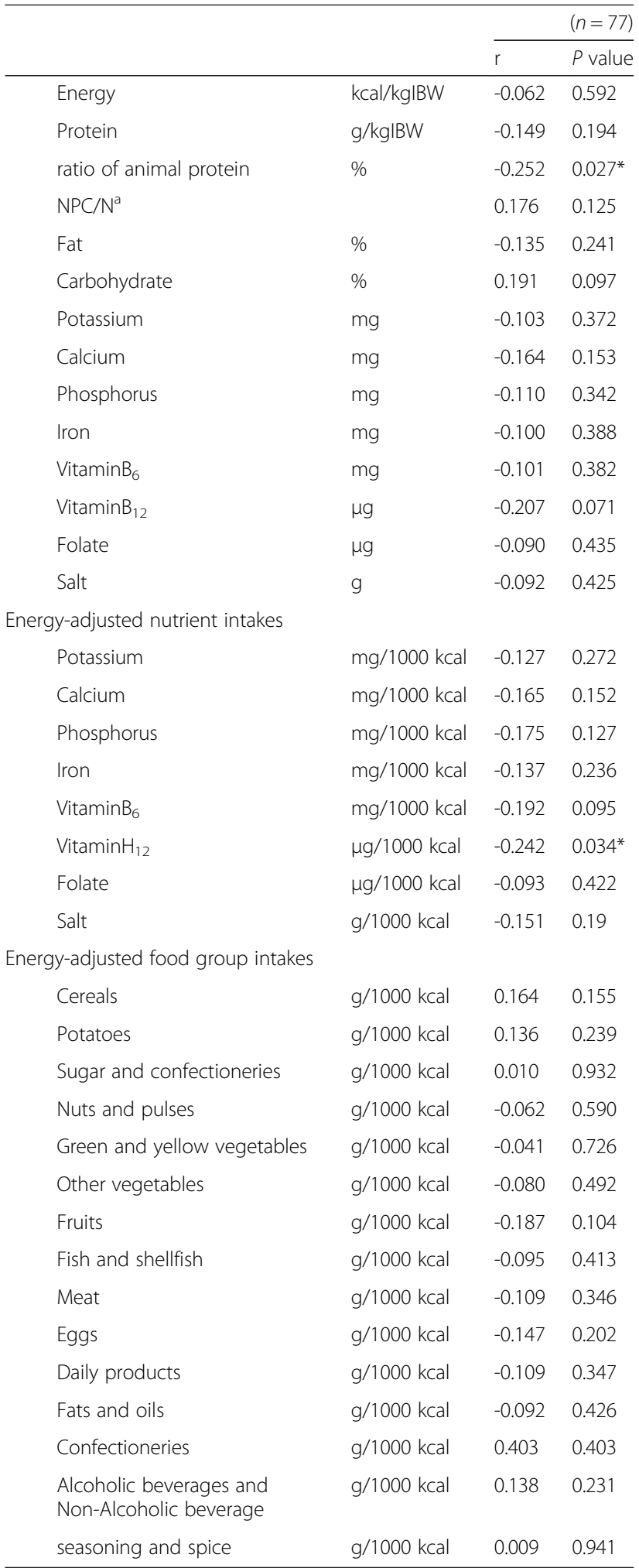

${ }^{a} N P C / N$, ratio of non-protein energy per nitrogen

Data not regularly distributed were log transformed for further statistical analysis. ${ }^{*} p<0.05$

Adjusted for gender, age which association was noted as response variables. The results are shown in Table 7.

On analysis using age and sex as adjustment factors, factors influencing the serum Hcy level were the serum $\mathrm{VB}_{12}$, folate, and IP levels as well as animal protein intake ratio and serum Alb level. The results in the analysis of variance table was significant $(P<0.001)$ with $R^{2}=0.552$ and adjusted $R^{2}=0.513$. The Durbin-Watson ratio was 1.716 , being non-problematic, and there was no outlying predicted value exceeding $\pm 3 S D$ of the measured value.

\section{Discussion}

In this study, the actual state of nutritional management of long-term hemodialysis patients was investigated at first. Nutritional management contributed to the maintenance of a high quality of life for these long-term hemodialysis patients, i.e., high level of independence in ADL was maintained. In particular, it was clarified that the influence of MTHFR C677T polymorphism-which has attracted international attention as a risk gene for cardiovascular disease that were major reasons for poor outcome in hemodialysis patient could be overcome. Regarding nutritional condition, mean BMI was $19.7 \pm$ $2.7 \mathrm{~kg} / \mathrm{m}^{2}$, mean serum Alb level was $3.7 \pm 0.3 \mathrm{~g} / \mathrm{dL}$, and median GNRI was $93 \pm 6$. Since the reported BMI of Japanese hemodialysis patients is $21.4 \pm 4.1 \mathrm{~kg} / \mathrm{m}^{2}$ [5], their nutritional condition was favorable.

The frequencies of CC, CT, and TT genotypes of MTHFR C677T were 29.5, 43.6, and $26.9 \%$, respectively, and the frequency of TT genotype was significantly higher than the frequency of that in Japanese hemodialysis patients as reported by Morimoto et al. (13.7 \%) [11] and Kimura et al. (17.4\%) [22] and healthy Japanese (about $15 \%)$ [23, 24]. Many studies reported that MTHFR C677T polymorphism is a risk factor for nephropathy, and a significantly high frequency of the TT genotype in nephropathy patients was also detected in a meta-analysis conducted by Yang et al. [25]. Similar results were obtained in studies of Asians reported by Sun et al. [26] and Mtiraoui et al. [27]. In studies of Japanese hemodialysis patients, Kimura et al. [22] investigated the association between MTHFR C677T and hyperhomocysteinemia, and Morimoto et al. [11] investigated MTHFR C677T, hyperhomocysteinemia, and risk of cardiovascular disease, but they did not investigate whether the polymorphism is a risk factor for nephropathy. However, they investigated the genotype frequency of MTHFR C677T in hemodialysis patients, and the frequency of the TT genotype was similar to those reported by Sun et al. [26] and Mtiraoui et al. [27]. The TT genotype have been reported as a risk factor for cardiovascular disease by Morimoto et al. [11], and for death in end-stage renal failure patients by Jamison et al. [28], which cannot be explained by the results of our study. It was assumed that 
Table 7 Multiple regression analyses to test the effects of serum components on serum homocysteine concentration

\begin{tabular}{|c|c|c|c|c|c|}
\hline \multicolumn{6}{|l|}{ Response variable; serum Hcy } \\
\hline \multirow{2}{*}{$\begin{array}{l}\text { Explanatory variable } \\
\text { Serum albumin }(\mathrm{g} / \mathrm{dL})\end{array}$} & Standardized partial regression coefficient ( $\beta$ ) & \multirow{2}{*}{$\begin{array}{c}\text { Standard error } \\
0.048\end{array}$} & \multirow{2}{*}{$\begin{array}{c}P \text { value } \\
0.007\end{array}$} & \multicolumn{2}{|c|}{$95 \%$ confidential interval } \\
\hline & 0.228 & & & 0.037 & 0.229 \\
\hline Serum inorganic phosphorus (mg/dL) & 0.242 & 0.012 & 0.006 & 0.009 & 0.056 \\
\hline Serum folate (ng/mL) & -0.351 & 0.007 & $<0.0001$ & -0.043 & -0.015 \\
\hline Serum Vitamin $\mathrm{B}_{12}(\mathrm{pmol} / \mathrm{L})$ & -0.367 & 0.0001 & $<0.0001$ & 0 & 0 \\
\hline Ratio of animal protein (\%) & -0.213 & 0.001 & 0.016 & -0.005 & -0.001 \\
\hline
\end{tabular}

the frequency of the TT genotype at the time of introduction of dialysis was higher than the reported frequency because the duration of dialysis therapy was 7-10 years in the reports described above, which was far shorter than that in our study. Serum folate (5-methyltetrahydrofolate) decreases with the TT genotype because of a $70 \%$ decrease in the MTHFR enzyme activity level compared with the activity with the $\mathrm{CC}$ genotype, which inhibits the pathway of conversion of homocysteine to methionine and elevates the serum Hcy level. Hyperhomocysteinemia promotes renal dysfunction, as reported by Wollesen et al. [29]. It has also been reported that the prevalence of hyperhomocysteinemia is high in hemodialysis patients, and hyperhomocysteinemia is also a risk factor for death. Similarly, hyperhomocysteinemia was noted in most patients (96\%) in our study. The physical condition, biochemical parameters, and dietary intake were compared among the MTHFR C677T genotypes to clarify the influence of this polymorphism. No significant differences in age, duration of dialysis therapy, age at introduction of dialysis, BMI, serum Alb level, or GNRI among different genotypes were noted. BADL was also not significantly different, and the percentages of independent patients were high with each genotype. Two of three patients "requiring assistance" harbored the TT genotype, but as described above, they were elderly, the duration of dialysis therapy was long (38 years) and they were independent in their activities of daily living, although some patients used a wheelchair. There were no significant differences in nutrient or food group intake among the different genotypes. The energy, protein, potassium, and phosphorus intakes met the nutritional levels recommended in the guidelines in all three genotypes. The salt intake of patients markedly exceeded the amount specified in the guidelines, indicating excessive consumption. However, their body weight was controlled, based on the examination of body weight changes between dialysis sessions. As reported by several preceding studies, the serum folate level was low and the serum Hcy level was high with the TT genotype. In addition, folate and vitamin $\mathrm{B}_{12}$ intakes met the Dietary Reference Intakes of Japanese dietary recommendations, while vitamin $\mathrm{B}_{6}$ was slightly insufficient. Based on these findings, although the serum folate and Hcy levels were influenced by the genetic polymorphism, nutritional management was appropriate.

Four limitations of this study exist. First, the sample size was small, and investigation of sex differences was not performed. Second, the causal relationships between the factors influencing the serum Hcy level could not be identified because this was a cross-sectional study. Third, the dietary survey was capable of investigating habitual food intake over 1 month, but it is unclear whether the same pattern of food intake was maintained over a long period after introduction of dialysis. Fourth, the study results are not applicable to the present hemodialysis patients in Japan because diabetic nephropathy patients were absent in this study. Fifth, this is not a comparative study, the impact of some items that investigated in this study cannot be able to evaluate exactly. This study might be just a presentation of clinical and genetic profile of Japanese long-term dialysis patients.

A positive result was obtained in most studies that examined the association between serum Hcy level and arteriosclerotic disease. It has been clarified that hyperhomocysteinemia is a predictive factor for high mortality that is independent of other risk factors in coronary arterial disease patients [30]. Hyperhomocysteinemia was shown to be an independent risk factor in a study of 750 vascular disease patients at 19 institutions in 9 European countries [31]. It was also shown to be a strong cardiovascular disease risk factor in non-insulin dependent diabetes patients [32]. These findings are of interest with regard to the synergistic effect of risk factors. Inverse correlations of folate and vitamin $B_{12}$ levels with serum Hcy level in healthy subjects [23] have been reported, as well as the decrease in serum Hcy level after increased ingestion of these vitamins, but it remains to be investigated whether these findings are applicable to hemodialysis patients. 


\section{Conclusion}

In conclusion, it is natural that the frequency of the TT genotype of MTHFR C677T was very high (26.9\%) in patients because the TT genotype is a risk factor for renal failure and there might be many patients with the TT genotype at the time of introduction of dialysis. However, the frequency of the TT genotype was not high in long-term dialysis patients in preceding studies because the mortality rate during dialysis therapy was high with the TT genotype. The results of our study suggested that appropriate nutritional management decreases the high mortality rate in patients with the TT genotype.

\section{Competing interests}

This study was supported by a grant from Japanese Association of Dialysis Physicians.

\section{Authors' contributions \\ KS and YKan visited each facilities, and collected questionnaires and blood samples. MH and YKag carried out RFLP analysis. MH evaluated clinical data of patients. YKon evaluated and analyzed BDHQ food questionnaires. All authors read and approved the final manuscript.}

\begin{abstract}
Acknowledgement
The authors gratefully thank to the physicians and all medical staff in dialysis facilities as follow, Yoshizawa lin (Mamoru Yoshizawa), Ikenaga Jin Clinic (Hideki Ikenaga), Kameido Nephrology Clinic (Yoshiaki Itaya), Fureai Machida Hospital (Waichi Kitajima), Bosei Hospital (Tateki Kitaoka, Tetsuo Shirai, Kyoko Kino), Meguro Building Clinic (Tsuneo Takenaka), Matsumoto Clinic (Go Matsumoto, Takaomi Tanaka), Yotsuya Jin Clinic (Akiko Iwata), Namikibash Clinic (Masaru Ogawa), Kikuna Memorial Clinic (Hideki Uchimura), Saitama Tsukinomori Clinic (Satoshi Kurihara, Naoaki Hayama), Kidney Clinic Setagaya (Shinya Suganuma), Tokorozawa Jin Clinic (Hiroshi Nagaura). A part of this study was presented in the 46th annual meeting of American Society of Nephrology (Philadelphia, 2014).
\end{abstract}

\section{Sources of support}

Fund from Japanese Association of Dialysis Physicians.

\section{Author details}

${ }^{1}$ Department of Clinical Nutrition and Dietetics, Kagawa Nutrition University, Sakado, Japan. ${ }^{2}$ Department of Nephrology, Tokyo Medical University, 6-7-1 Nishi-Shinjuku, Shinjuku, Tokyo 160-0023, Japan. ${ }^{3}$ Department of Nutrition, School of Nursing and Nutrition, Shukutoku University, Chiba, Japan. ${ }^{4}$ Apheresis and Dialysis Center, School of Medicine, Keio University, Tokyo, Japan. ${ }^{5}$ Department of Health and Nutrition, Faculty of Human Life Studies, University of Niigata Prefecture, Niigata, Japan. ${ }^{6}$ Department of Medical Chemistry, Kagawa Nutrition University, Sakado, Japan.

Received: 3 July 2015 Accepted: 24 September 2015

Published online: 24 November 2015

\section{References}

1. Pifer TB, McCullough KP, Port FK, Goodkin DA, Maroni BJ, Held PJ, et al. Mortality risk in hemodialysis patients and changes in nutritional indicators: DOPPS. Kidney Int. 2002;62:2238-45.

2. Fouque D, Kalantar-Zadeh K, Kopple J, Cano N, Chauveau P, Cuppari L, et al. A proposed nomenclature and diagnostic criteria for protein-energy wasting in acute and chronic kidney disease. Kidney Int. 2008;73:391-8.

3. de Mutsert R, Snijder MB, van der Sman-de Beer F, Seidell JC, Boeschoten EW, Krediet RT, et al. Association between body mass index and mortality is similar in the hemodialysis population and the general population at high age and equal duration of follow-up. J Am Soc Nephrol. 2007;18:967-74.

4. Iseki K, Kawazoe N, Fukiyama K. Serum albumin is a strong predictor of death in chronic dialysis patients. Kidney Int. 1993;44:115-9.

5. Leavey SF, McCullough K, Hecking E, Goodkin D, Port FK, Young EW. Body mass index and mortality in 'healthier' as compared with 'sicker' haemodialysis patients: results from the Dialysis Outcomes and Practice Patterns Study (DOPPS). Nephrol Dial Transplant. 2001;16:2386-94.

6. Nakai S, Watanabe Y, Masakane I, Wada A, Shoji T, Hasegawa T, et al. Overview of regular dialysis treatment in Japan (as of 31 December 2011). Therap Apher Dial. 2013;17:567-611.

7. Bachmann J, Tepel M, Raidt H, Riezler R, Graefe U, Langer K, et al. Hyperhomocysteinemia and the risk for vascular disease in hemodialysis patients. J Am Soc Nephrol. 1995;6:121-5.

8. Frosst P, Blom HJ, Milos R, Goyette P, Sheppard CA, Matthews RG, et al. A candidate genetic risk factor for vascular disease: a common mutation in methylenetetrahydrofolate reductase. Nat Genet. 1995;10:111-3.

9. Morita H, Taguchi J, Kurihara H, Kitaoka M, Kaneda H, Kurihara Y, et al. Genetic polymorphism of 5, 10-methylenetetrahydrofolate reductase (MTHFR) as a risk factor for coronary artery disease. Circulation. 1997;95:2032-6.

10. Fodinger M, Mannhalter C, Wolfl G, Pabinger I, Muller E, Schmid R, et al. Mutation ( 677 C to T) in the methylenetetrahydrofolate reductase gene aggravates hyperhomocysteinemia in hemodialysis patients. Kidney Int. 1997:52:517-23.

11. Morimoto K, Haneda T, Okamoto K, Ishida H, Kikuchi K. Methylenetetrahydrofolate reductase gene polymorphism, hyperhomocysteinemia, and cardiovascular diseases in chronic hemodialysis patients. Nephron. 2002;90:43-50.

12. Sasaki S, Katagiri A, Tsuji T, Shimoda T, Amano K. Self-reported rate of eating correlates with body mass index in 18-y-old Japanese women. Int J Obes Relat Metab Disord. 2003;27:1405-10.

13. Okubo H, Sasaki S, Rafamantanantsoa HH, Ishikawa-Takata K, Okazaki H, Tabata I. Validation of self-reported energy intake by a self-administered diet history questionnaire using the doubly labeled water method in 140 Japanese adults. Eur J Clin Nutr. 2008;62:1343-50.

14. Shinzato T, Nakai S, Fujita Y, Takai I, Morita H, Nakane K, et al. Determination of Kt $\mathrm{N}$ and protein catabolic rate using pre- and postdialysis blood urea nitrogen concentrations. Nephron. 1994;67:280-90.

15. Kobayashi S, Honda S, Murakami K, Sasaki S, Okubo H, Hirota N, et al. Both comprehensive and brief self-administered diet history questionnaires satisfactorily rank nutrient intakes in Japanese adults. J Epidemiol. 2012;22:151-9.

16. Kobayashi S, Murakami K, Sasaki S, Okubo H, Hirota N, Notsu A, et al. Comparison of relative validity of food group intakes estimated by comprehensive and brief-type self-administered diet history questionnaires against $16 \mathrm{~d}$ dietary records in Japanese adults. Public Health Nutr. 2011;14:1200-11.

17. Sasaki S, Yanagibori R, Amano K. Self-administered diet history questionnaire developed for health education: a relative validation of the test-version by comparison with 3-day diet record in women. J Epidemiol. 1998;8:203-15.

18. Mahoney FI, Barthel DW. Functional evaluation: the Barthel index. Md State Med J. 1965;14:61-5.

19. Bouillanne O, Morineau G, Dupont C, Coulombel I, Vincent JP, Nicolis I, et al. Geriatric nutritional risk index: a new index for evaluating at-risk elderly medical patients. Am J Clin Nutr. 2005;82:777-83.

20. Yamada K, Furuya R, Takita T, Maruyama Y, Yamaguchi Y, Ohkawa S, et al. Simplified nutritional screening tools for patients on maintenance hemodialysis. Am J Clin Nutr. 2008;87:106-13.

21. Nakao T, Sanaka T, Tsubakihara Y, Hattori M, Honda M, Mizuiri S, et al. Dietary recommendations for chronic kidney disease, 2007. Jpn J Nephrol. 2007:49:871-8

22. Kimura H, Gejyo F, Suzuki S, Miyazaki R. The C677T methylenetetrahydrofolate reductase gene mutation in hemodialysis patients. J Am Soc Nephrol. 2000;11:885-93.

23. Hiraoka M, Kato K, Saito Y, Yasuda K, Kagawa Y. Gene-nutrient and genegene interactions of controlled folate intake by Japanese women. Biochem Biophys Research Commun. 2004;316:1210-6.

24. Sadewa AH, Sunarti, Sutomo R, Hayashi C, Lee MJ, Ayaki H, et al. The C677T mutation in the methylenetetrahydrofolate reductase gene among the Indonesian Javanese population. Kobe J Med Sci. 2002;48:137-44.

25. Yang S, Zhang J, Feng C, Huang G. MTHFR 677T variant contributes to diabetic nephropathy risk in Caucasian individuals with type 2 diabetes: a meta-analysis. Metabolism. 2013;62:586-94.

26. Sun J, Xu Y, Zhu Y, Lu H. Genetic polymorphism of methylenetetrahydrofolate reductase as a risk factor for diabetic nephropathy in Chinese type 2 diabetic patients. Diabetes Res Clin Pract. 2004;64:185-90.

27. Mtiraoui N, Ezzidi I, Chaieb M, Marmouche H, Aouni Z, Chaieb A, et al. MTHFR C677T and A1298C gene polymorphisms and 
hyperhomocysteinemia as risk factors of diabetic nephropathy in type 2 diabetes patients. Diabetes Res Clin Pract. 2007;75:99-106.

28. Jamison RL, Shih MC, Humphries DE, Guarino PD, Kaufman JS, Goldfarb DS, et al. Effect of the MTHFR C677T and A1298C polymorphisms on survival in patients with advanced CKD and ESRD: a prospective study. Am J Kidney Dis. 2009;53:779-89.

29. Wollesen F, Brattstrom L, Refsum H, Ueland PM, Berglund L, Berne C. Plasma total homocysteine and cysteine in relation to glomerular filtration rate in diabetes mellitus. Kidney Int. 1999:55:1028-35.

30. Nygard O, Nordrehaug JE, Refsum H, Ueland PM, Farstad M, Vollset SE. Plasma homocysteine levels and mortality in patients with coronary artery disease. N Engl J Med. 1997;337:230-6.

31. Graham IM, Daly LE, Refsum HM, Robinson K, Brattstrom LE, Ueland PM, et al. Plasma homocysteine as a risk factor for vascular disease. The European Concerted Action Project. JAMA. 1997:277:1775-81.

32. Hoogeveen EK, Kostense PJ, Beks PJ, Mackaay AJ, Jakobs C, Bouter LM, et al. Hyperhomocysteinemia is associated with an increased risk of cardiovascular disease, especially in non-insulin-dependent diabetes mellitus: a populationbased study. Arterioscler Thromb Vasc Biol. 1998;18:133-8.

\section{Submit your next manuscript to BioMed Central and take full advantage of:}

- Convenient online submission

- Thorough peer review

- No space constraints or color figure charges

- Immediate publication on acceptance

- Inclusion in PubMed, CAS, Scopus and Google Scholar

- Research which is freely available for redistribution 